\title{
THERMODYNAMIC STABILITY OF TWO RHOMBOHEDRAL PHASES OF $\mathrm{Si}_{0.5} \mathrm{Ge}_{0.5}$ ALLOY
}

\author{
P. Boguslawski \\ Institute of Physics, Polish Academy of Sciences \\ Al. Lotników 32/46, 02-668 Warszawa, Poland
}

\begin{abstract}
Thermodynamic stability of two ordered phases, RS1 and RS2, of $\mathrm{Si}_{0.5} \mathrm{Ge}_{0.5}$ alloy is considered. Bulk and surface formation enthalpies are calculated using the model Tersoff's potential. RS2 structure is unstable, but its (001) ordered surface is stable against segregation. Properties of RS1 are just the opposite.
\end{abstract}

PACS numbers: $61.55 . \mathrm{Hg}, 68.35 . \mathrm{Md}, 68.60 . \mathrm{Dv}$

The present work is motivated by recent experimental studies on spontaneous ordering of $\mathrm{Si}_{0.5} \mathrm{Ge}_{0.5}$ alloy [1-7]. The order takes the form of $\mathrm{Si}_{2} / \mathrm{Ge}_{2}$ superlattice oriented in $\{111\}$ directions. This structure has the rhombohedral symmetry, and may exist in two variants shown in Fig. 1. In the first one, denoted by RS1, the widely spaced (111) planes are occupied by the same type of atoms forming a sequence $-\mathrm{Si} \equiv \mathrm{Ge}-\mathrm{Ge} \equiv \mathrm{Si}-. .$. , where the dashes denote bonds between planes. In the second RS2 variant the widely spaced (111) planes are occupied by unlike atoms, forming a sequence $-\mathrm{Si} \equiv \mathrm{Si}-\mathrm{Ge} \equiv \mathrm{Ge}-$... Analysis by LeGoues et al. [4] has demonstrated that the phase that grows spontaneously is the RS2 structure. Upon annealing this phase disappears, and the alloy becomes random. However, in the case of $\mathrm{Si} / \mathrm{SiGe}$ or $\mathrm{Si} / \mathrm{Ge}$ superlattices, a long annealing may lead to a reversible appearance of the long-range order at the interfaces, which now takes the form of the RS1 structure [7].

Here, we present an attempt to understand this behavior, and we analyze thermodynamic stability at $T=0$ of $\mathrm{Si}_{0.5} \mathrm{Ge}_{0.5}$ alloy. Stability of the phase $\alpha$ against segregation into pure constituents is given by the formation enthalpy:

$$
\Delta H=E^{\alpha}-\frac{1}{2}\left[E^{\mathrm{Si}}+E^{\mathrm{Ge}}\right],
$$

where $E^{\mathrm{X}}$ is the total energy per atom for $\mathrm{X}(=\alpha, \mathrm{Si}, \mathrm{Ge})$ at equilibrium. In the case of epitaxial growth, when the lattice constant of the epilayer in the plane of growth is equal to that of the substrate, the formation enthalpy takes the value

$$
\Delta H_{\mathrm{epi}}=E_{\mathrm{epi}}^{\alpha}-\frac{1}{2}\left[E_{\mathrm{epi}}^{\mathrm{Si}}+E_{\mathrm{epi}}^{\mathrm{Ge}}\right],
$$




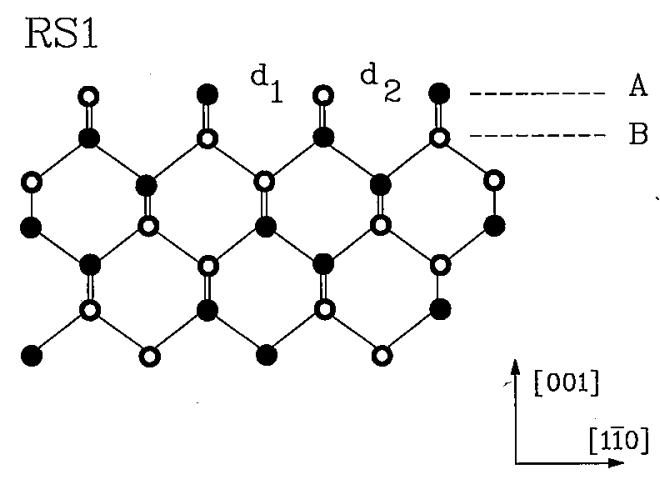

RS2

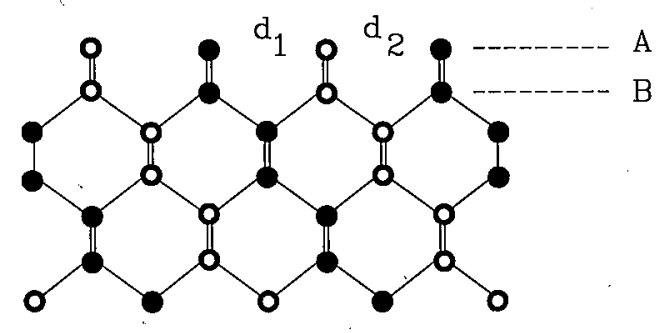

Fig. 1. RS1 and RS2 structures projected on (110) plane. Open circles represent Ge columns, and solid circles Si columns. Surfaces of type A and B are shown. $d_{1}$ and $d_{2}$ indicate two pairs of atoms forming non-equivalent dimers at the surface $A$.

where $E_{\text {epi }}^{\mathrm{X}}$ is the total energy of epitaxial X $(=\alpha, \mathrm{Si}, \mathrm{Ge})$. Finally, we shall also investigate stability of surfaces terminating $\mathrm{Si}_{0.5} \mathrm{Ge}_{0.5}$ alloys against two-dimensional segregation at the surface given by formation enthalpy:

$$
\Delta H_{\text {sur }}=E^{\alpha}[n]-\frac{1}{2}\left\{E^{\alpha}[n-1 ; \mathrm{Si}]+E^{\alpha}[n-1 ; \mathrm{Ge}]\right\},
$$

where $E^{\alpha}[n]$ is the total energy of the substrate covered by $n$ layers of alloy in the phase $\alpha$, and e.g. $E^{\alpha}[n-1$; Si] is the total energy of $(n-1)$ layers of the phase $\alpha$ terminated by a $\mathrm{Si}$ layer at the surface.

Total energies of the considered systems are calculated by using the model interatomic potential proposed by Tersoff [8]. This potential takes into account not only the elastic energy of bonds deformed by the lattice mismatch of $\mathrm{Si}_{0.5} \mathrm{Ge}_{0.5}$ alloy, but also the chemical energy induced by a formation of heteropolar $\mathrm{Si}-\mathrm{Ge}$ bonds [9]. Pseudomorphic growth on (001) Si substrate was assumed.

The calculated formation enthalpies are given in the Table. The good agreement of $\Delta H$ and $\Delta H_{\text {epi }}$ for RS1 with the results of ab initio calculations $[10,11]$ demonstrates that the chemical contribution to the formation enthalpy is well described by the Tersoff's potential.

Free (001) surfaces of both $\mathrm{Si}$ and Ge undergo a reconstruction - its most simple and most frequent form is the $2 \times 1$ dimerized surface. We find that the 
equilibrium configuration is the symmetric dimer with energy of dimerization $E_{\text {dim }}=1.44$ and $1.15 \mathrm{eV}$ per dimer for $\mathrm{Si}$ and $\mathrm{Ge}$, respectively, in reasonable agreement with literature $[12,13]$.

\section{TABLE}

Calculated formation enthalpies for RS1 and RS2 phases of $\mathrm{Si}_{0.5} \mathrm{Ge}_{0.5}$ ordered alloy. $\Delta H$ and $\Delta H_{\text {epi }}$ are in $\mathrm{meV} /$ atom, $\Delta H_{\text {sur }}$ and $E_{\text {dim }}$ are in $\mathrm{meV} /$ dimer and $\mathrm{eV} /$ dimer, respectively. In parentheses, the type of the surface is given.

\begin{tabular}{l|r|r|r|r}
\hline \hline Phase & $\Delta H$ & $\Delta H_{\text {epi }}$ & $\Delta H_{\text {sur }}$ & $E_{\text {dim }}$ \\
\hline RS1 & 7 & -4 & 0 & $1.326(\mathrm{~A})$ \\
& & & +19 & $1.332(\mathrm{~B})$ \\
RS2 & 12 & 1 & -4 & $1.374(\mathrm{~A})$ \\
& & & -5 & $1.338(\mathrm{~B})$
\end{tabular}

The obtained results may be summarized as follows:

(i) In pure $\mathrm{Si}$ or Ge, any two consecutive surface layers with $2 \times 1$ and $1 \times 2$ reconstruction are physically equivalent, since they differ by a $90^{\circ}$ rotation. In contrast, for both RS1 and RS2 there are two non-equivalent surfaces, labeled A and B in Fig. 1. E.g., in the case of the surface B of RS2, each surface atom makes one homobond and one heterobond with subsurface atoms, and each surface dimer is a homodimer. This surface has the $2 \times 2$ periodicity, which should be observed by e.g. RHEED. For the surface $A$ of RS2, each atom makes two homobonds with underlying atoms, the dimers are heterodimers, and the periodicity is $2 \times 1$. In consequence of this non-equivalence, surface formation enthalpies and energies of dimerization are different for surfaces of type A and B, see Table. Moreover, for A-type surfaces there are two possible dimerization patterns. In the case of RS2, the $d_{1}$ dimer of Fig. 1 is more stable by as much as $127 \mathrm{meV}$ per dimer than the dimer $d_{2}$, while for RS1 the difference is much smaller, amounting to about $5 \mathrm{meV}$ per dimer.

(ii) The RS1 structure is unstable in the free case, since $\Delta H^{\mathrm{RS} 1}=7 \mathrm{meV} /$ atom. However, this phase becomes substrate-stabilized, as we find $\Delta H_{\text {epi }}<0$, which may explain the observed reversible ordering in this phase. On the other hand, its surface is unstable (which should prevent the two-dimensional ordering at the surface during epitaxial growth). As pointed out by Mäder et al. [14], there is also an interface-specific contribution to formation enthalpy which drives the reversible ordering at $\mathrm{Si} / \mathrm{Ge}$ interfaces.

(iii) Properties of RS2 are just the opposite to those of RS1. Namely, bulk RS2 is found to be unstable, both in the free and in the epitaxial forms. This explains the observed randomization of this phase. On the other hand, the 
(001) surface of the RS2 phase is stable against surface segregation. This conditions the epitaxial growth of this phase, and confirms the proposition that the spontaneous ordering is a surface process occurring during epitaxy. One may notice that the spontaneous ordering in the form of (111) superlattices, which are intrinsically unstable but terminated by stable surfaces, has been recently observed for III-V alloys [15].

(iv) For non-dimerized surfaces $\mathrm{A}$ and $\mathrm{B}$ of $\mathrm{RS2}$ we obtain $\Delta H_{\text {sur }}=-7$ and $-16 \mathrm{meV} /$ dimer, respectively. These values are comparable to those for dimerized surfaces, which are given in the Table.

The author would like to acknowledge the hospitality of Prof. A. Baldereschi from IRRMA, Lausanne, where a part of the calculations has been done.

\section{References}

[1] A. Ourmazd, J.C. Bean, Phys. Rev. Lett. 55, 765 (1985).

[2] D.J. Lockwood, K. Rajan, E.W. Fenton, J.-M. Baribeau, M.W. Denhoff, Solid State Commun. 61, 465 (1987).

[3] E. Muller, H.-U. Nissen, M. Ospelt, H. von Känel, Phys. Rev. Lett. 63, 1819 (1989).

[4] F.K. LeGoues, V.P. Kesan, S.S. Iyer, Phys. Rev. Lett. 64, 40 (1990).

[5] F.K. LeGoues, V.P. Kesan, S.S. Iyer, J. Tersoff, R. Tromp, Phys. Rev. Lett. 64, 2038 (1990).

[6] N. Ohshima et al., Appl. Phys. Lett. 57, 2434 (1990).

[7] E. Muller, H.-U. Nissen, K. Mäder, M. Ospelt, H. von Kanel, to be published.

[8] J. Tersoff, Phys. Rev. B 39, 5566 (1989).

[9] P.B. Littlewood, Phys. Rev. B 34, 1363 (1986), and B. Koiler and M.O. Robbins, Phys. Rev. B 40, 12554 (1989) have investigated the stability of Si/Ge alloys using the valence-force-field model, which neglects the charge transfer energy.

[10] J.L. Martins, A. Zunger, Phys. Rev. Lett. 56, 1400 (1986).

[11] S. Ciraci, I.P. Batra, Phys. Rev. Lett. 58, 2114 (1987).

[12] K.C. Pandey, Proc. 17 ICPS, Eds. J.D. Chadi, W.A. Harrison, Springer-Verlag, New York 1985, p. 55

[13] I.P. Batra, Phys. Rev. B 41, 5048 (1990).

[14] K.A. Mäder, H. von Känel, A. Baldereschi, Microstruct. and Superlattices, in print.

[15] P. Boguslawski, Phys. Rev. B 42, 3737 (1990). 